\title{
An analysis of tumor-related potential spinal column instability (Spine Instability Neoplastic Scores 7-12) eventually requiring surgery with a 1-year follow-up
}

\author{
Enrique Vargas, BS, ${ }^{1,4}$ Dennis T. Lockney, MD, ${ }^{1}$ Praveen V. Mummaneni, MD, MBA, ${ }^{1}$ \\ Alexander F. Haddad, BS, ${ }^{1,4}$ Joshua Rivera, BA, ${ }^{1}$ Xiao Tan, BA, ${ }^{1}$ Alysha Jamieson, BA, ${ }^{1}$ \\ Yasmine Mahmoudieh, BA, ${ }^{1}$ Sigurd Berven, MD, ${ }^{3}$ Steve E. Braunstein, MD, PhD, ${ }^{2}$ and \\ Dean Chou, MD'
}

Departments of ${ }^{1}$ Neurosurgery, ${ }^{2}$ Radiation Oncology, and ${ }^{3}$ Orthopedic Surgery, and ${ }^{4}$ School of Medicine, University of California, San Francisco, California

OBJECTIVE Within the Spine Instability Neoplastic Score (SINS) classification, tumor-related potential spinal instability (SINS 7-12) may not have a clear treatment approach. The authors aimed to examine the proportion of patients in this indeterminate zone who later required surgical stabilization after initial nonoperative management. By studying this patient population, they sought to determine if a clear SINS cutoff existed whereby the spine is potentially unstable due to a lesion and would be more likely to require stabilization.

METHODS Records from patients treated at the University of California, San Francisco, for metastatic spine disease from 2005 to 2019 were retrospectively reviewed. Seventy-five patients with tumor-related potential spinal instability (SINS 7-12) who were initially treated nonoperatively were included. All patients had at least a 1-year follow-up with complete medical records. A univariate chi-square test and Student t-test were used to compare categorical and continuous outcomes, respectively, between patients who ultimately underwent surgery and those who did not. A backward likelihood multivariate binary logistic regression model was used to investigate the relationship between clinical characteristics and surgical intervention. Recursive partitioning analysis (RPA) and single-variable logistic regression were performed as a function of SINS.

RESULTS Seventy-five patients with a total of 292 spinal metastatic sites were included in this study; 26 (34.7\%) patients underwent surgical intervention, and 49 (65.3\%) did not. There was no difference in age, sex, comorbidities, or lesion location between the groups. However, there were more patients with a SINS of 12 in the surgery group (55.2\%) than in the no surgery group $(44.8 \%)(p=0.003)$. On multivariate analysis, $\operatorname{SINS}>11(\mathrm{OR} 8.09, \mathrm{Cl} 1.96-33.4, \mathrm{p}=$ 0.004 ) and Karnofsky Performance Scale (KPS) score < 60 (OR 0.94, Cl 0.89-0.98, p = 0.008) were associated with an increased risk of surgery. KPS score was not correlated with SINS $(p=0.4)$. RPA by each spinal lesion identified an optimal cutoff value of SINS > 10, which were associated with an increased risk of surgical intervention. Patients with a surgical intervention had a higher incidence of complications on multivariable analysis (OR 2.96, $\mathrm{Cl} 1.01-8.71, p=0.048)$.

CONCLUSIONS Patients with a mean SINS of 11 or greater may be at increased risk of mechanical instability requiring surgery after initial nonoperative management. RPA showed that patients with a KPS score of 60 or lower and a SINS of greater than 10 had increased surgery rates.

https://thejns.org/doi/abs/10.3171/2021.2.FOCUS201098

KEYWORDS Spine Instability Neoplastic Score; SINS; tumor

A LONG with the advent of the Patchell ${ }^{1}$ criteria and NOMS $^{2}$ framework to guide indications for surgery in metastatic spine tumor patients, the Spine Instability Neoplastic Score (SINS) has also helped to determine the stability of the spine. ${ }^{2,3}$ SINS characterizes the stability of the spine with metastatic tumors. ${ }^{3,4}$ Scores of 0-6 are considered to indicate spinal stability, those of 7-12 indicate potential instability, and those of 13-18 are defined as unstable. However, a paucity of literature exists regarding the optimal treatment of patients in the potentially unstable (scores 7-12) category.

SINS has been instrumental in assessing spinal stabil-

ABBREVIATIONS $\mathrm{BED}_{3}=$ biologically effective dose; $\mathrm{CCI}=$ Charlson Comorbidity Index; KPS = Karnofsky Performance Scale; RPA = recursive partitioning analysis; SINS = Spine Instability Neoplastic Score. 
TABLE 1. Treatment, acute outcomes, and backward likelihood binary logistic regression for surgery in patients initially treated with radiation

\begin{tabular}{|c|c|c|c|c|}
\hline Variable & All Patients $(n=75)$ & No Surgery $(n=49)$ & Surgery $(n=26)$ & p Value \\
\hline Chemotherapy & $15(20.0 \%)$ & $3(6.1 \%)$ & $12(46.2 \%)$ & $<0.001$ \\
\hline Mean $\mathrm{BED}_{3}$ radiation dosing $(\mathrm{SD})$ & $56.1(30.1)$ & $53.1(30.3)$ & $63.12(30.5)$ & 0.181 \\
\hline Kyphoplasty & $3(4.0 \%)$ & $0(0.0 \%)$ & $3(11.5 \%)$ & 0.015 \\
\hline Vertebroplasty & $3(4.0 \%)$ & $0(0.0 \%)$ & $3(11.5 \%)$ & 0.015 \\
\hline Any peri-admission complication & $20(26.7 \%)$ & $9(18.4 \%)$ & $11(42.3 \%)$ & 0.026 \\
\hline 30-day readmission & $5(6.7 \%)$ & $1(2.0 \%)$ & $4(15.4 \%)$ & 0.046 \\
\hline 90-day readmission & $7(9.3 \%)$ & $1(2.0 \%)$ & $6(23.1 \%)$ & 0.006 \\
\hline 30-day reoperation & $4(5.3 \%)$ & $0(0.0 \%)$ & $4(15.4 \%)$ & 0.012 \\
\hline 90-day reoperation & $4(5.3 \%)$ & $0(0.0 \%)$ & $4(15.4 \%)$ & 0.012 \\
\hline Variable & OR & \multicolumn{2}{|c|}{$95 \% \mathrm{Cl}$} & $\mathrm{p}$ Value \\
\hline SINS 11 & 3.86 & \multicolumn{2}{|c|}{$0.81-18.4$} & 0.090 \\
\hline SINS 12 & 8.09 & \multicolumn{2}{|c|}{$1.96-33.4$} & 0.004 \\
\hline KPS score & 0.94 & \multicolumn{2}{|c|}{$0.89-0.98$} & 0.008 \\
\hline
\end{tabular}

Values represent the number of patients (\%) unless stated otherwise.

ity as a validated metric ${ }^{5-7}$ with high inter-rater reliability among different medical specialties: ${ }^{8-12}$ it also has been seminal for assessing spinal stability in the setting of spinal metastases. The utility of SINS has been applied to other aspects of spine oncology care as well, such as predicting vertebral body fracture after radiotherapy, ${ }^{13,14}$ predicting mechanical pain/instability in patients with metastatic disease, ${ }^{15,16}$ and correlating SINS with prognosis. ${ }^{17,18}$ However, questions remain in the potentially unstable spine category, which includes patients with SINS of 7-12. Versteeg et al. stated, "Within the SINS potentially unstable group, distinct clinical profiles were observed in patients treated with surgery or radiotherapy alone." Although a general consensus exists for the treatment of lesions falling into the stable and unstable SINS categories, the potentially unstable category encompasses a wide range of lesions and patients, with variation in treatment strategies. We evaluated patients with SINS of 7-12 and studied which patients ultimately underwent surgical stabilization. We attempted to identify a threshold score for surgical stabilization within this category of tumor-related potential spinal instability.

\section{Methods}

\section{Patient Population}

Adult patients who were diagnosed with metastatic spine disease from June 2005 to November 2019 at the University of California, San Francisco, were retrospectively studied. Inclusion criteria were potential spinal column instability (SINS 7-12), initial nonoperative management (i.e., radiotherapy or chemotherapy), and a minimum 1-year follow-up. Exclusion criteria included incomplete medical records, patients who were initially treated with surgery followed by adjuvant therapy, lack of a minimum 1-year follow-up, and lack of proper imaging to calculate SINS.

\section{Data Collection}

Data were collected by four independent observers. Demographic variables included age and sex. Clinical variables included date of initial evaluation, date of preoperative chemotherapy, SINS score (location, pain, bone lesion, radiographic spinal alignment, vertebral body collapse, posterior spinal element involvement), primary tumor histology, and date of latest follow-up. Treatment variables included the date of initial radiation treatment, total radiation dose (in Gy), radiation dose per fraction, biologically effective dose $\left(\mathrm{BED}_{3}\right)$ to normal tissue, surgery date, and surgical procedure. Patients who required stabilization via kyphoplasty or vertebroplasty were included in the group requiring surgery. Physical status variables included the Charlson Comorbidity Index (CCI) score, Karnofsky Performance Scale (KPS) score, and the American Society of Anesthesiologists class. Complications, readmission rates, and revision surgeries were recorded for all patients (Table 1).

\section{Statistical Analysis}

The univariate chi-square test and Student t-test were used to compare categorical and continuous outcomes, respectively, between patients treated with surgery and without. A backward likelihood multivariate binary logistic regression model involving all variables with a $\mathrm{p}$ value $<0.20$ on univariate analysis was used to investigate the relationship between clinical characteristics and surgical intervention. KPS scores and SINS were initially included in the model. A similar model was utilized to assess the relationship between a perioperative complication and patient clinical characteristics. Variables initially incorporated into the model included patient age, $\mathrm{BED}_{3}$, and whether the patient had surgical intervention. A logistic regression model as a function of SINS was utilized to determine if there existed a SINS cutoff at which surgical intervention 


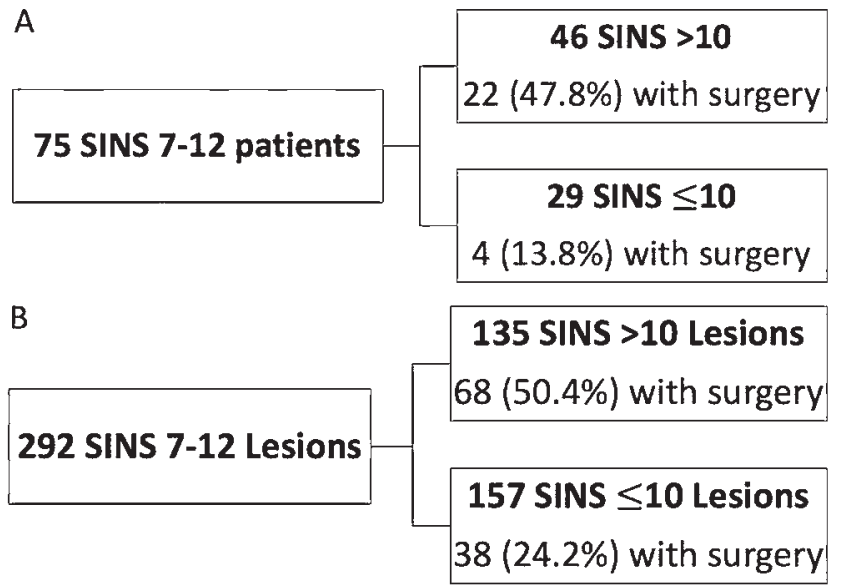

FIG. 1. RPA per patient (A) and per lesion (B).

was more probable than not. Recursive partitioning analysis (RPA) was also utilized to identify risk factors for surgical intervention and clinically relevant cutoffs. RPA was performed using per-patient (Fig. 1A) and per-lesion (Fig. 1B) data; $p<0.05$ was used as the threshold of statistical significance. All statistical analysis was performed using IBM SPSS Statistics (version 26; IBM Corp.).

\section{Results}

\section{SINS Scores and Primary Cancer Pathologies}

A total of 75 patients with 292 metastatic lesions were included in this study. SINSs of the patients were as follows: $3(4.0 \%)$ with SINS of 7, 7 (9.3\%) with SINS 8, 7 (9.3\%) with SINS 9, 12 (16.0\%) with SINS 10, 17 (22.7\%) with SINS 11, and 29 (38.7\%) with SINS 12 (Fig. 2). Patient and lesion SINSs can be found in Table 2. A number of different primary cancers were represented (Table 2), with breast cancer being the most common per patient $(20$, $26.7 \%$ ) and per lesion $(82,28.1 \%)$.

\section{Predictors of Surgical Intervention}

A comparison of clinical characteristics and demographics between patients with and without surgical intervention is shown in Table 3 . Of the 75 patients, 26 (34.7\%) underwent surgical intervention and 49 (65.3\%) did not. The average age was 59.1 years with no difference between patients with surgical intervention and those without (61.8 years vs 57.6 years, $\mathrm{p}=0.204)$. Similarly, there were no differences in patient sex, $\mathrm{BED}_{3}$, or CCI score between patients who underwent upfront surgery and those not requiring immediate stabilization. However, patients with a SINS of 12 were more likely to undergo surgical intervention than those who did not $(55.2 \%$ vs $44.8 \%, \mathrm{p}=0.004$, Table 1). Patients who underwent surgical intervention also had a lower median KPS score than those without surgical intervention (60 vs 70, $\mathrm{p}=0.001$ ). Specific SINS components that were significantly different between those requiring stabilization and those that did not included lesion type (lytic, mixed, or blastic) $(\mathrm{p}=$ 0.015 ) and extent of posterior element involvement (bilat-

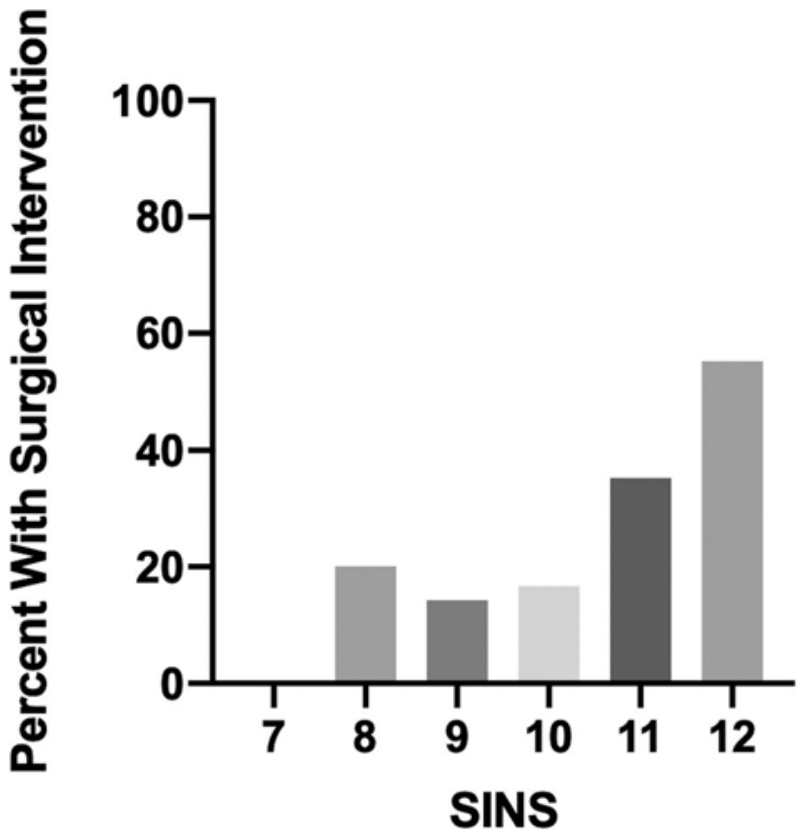

FIG. 2. Histogram of the percentages of patients who underwent surgery in the SINS 7-12 category.

TABLE 2. SINS and primary cancer pathologies

\begin{tabular}{|c|c|c|}
\hline & $\begin{array}{l}\text { No. of Patients (\%) } \\
\qquad(\mathrm{n}=75)\end{array}$ & $\begin{array}{l}\text { No. of Lesions }(\%) \\
\qquad(n=292)\end{array}$ \\
\hline \multicolumn{3}{|l|}{ SINS } \\
\hline 7 & $3(4.0)$ & $18(6.2)$ \\
\hline 8 & $7(9.3)$ & $25(8.6)$ \\
\hline 9 & $7(9.3)$ & $32(11.0)$ \\
\hline 10 & $12(16.0)$ & $82(28.1)$ \\
\hline 11 & $17(22.7)$ & $83(28.4)$ \\
\hline 12 & $29(38.7)$ & $52(17.8)$ \\
\hline \multicolumn{3}{|l|}{ Pathology } \\
\hline Salivary & $2(2.6)$ & $9(3.1)$ \\
\hline Kidney & $3(4.0)$ & $8(2.7)$ \\
\hline Breast & $20(26.7)$ & $82(28.1)$ \\
\hline Colorectal & $1(1.3)$ & $5(1.7)$ \\
\hline Lung & $10(13.3)$ & $36(12.3)$ \\
\hline Prostate & $17(22.7)$ & $66(22.6)$ \\
\hline Gastroesophageal & $1(1.3)$ & $4(1.4)$ \\
\hline Unknown GI & $1(1.3)$ & $7(2.4)$ \\
\hline Lymphoma & $1(1.3)$ & $4(1.4)$ \\
\hline Myeloma & $3(4.0)$ & $17(5.8)$ \\
\hline Melanoma & $3(4.0)$ & $8(2.7)$ \\
\hline Liver & $2(2.7)$ & $4(1.4)$ \\
\hline Pancreatic & $3(4.0)$ & $15(5.1)$ \\
\hline Chordoma & $3(4.0)$ & $7(2.4)$ \\
\hline Neuroblastoma & $2(2.7)$ & $6(2.1)$ \\
\hline Sarcoma & $1(1.3)$ & $6(2.1)$ \\
\hline
\end{tabular}


TABLE 3. Comparison of patients with and patients without stabilization surgery

\begin{tabular}{|c|c|c|c|c|}
\hline Variable & All Patients $(n=75)$ & No Surgery $(n=49)$ & Surgery $(n=26)$ & $p$ Value \\
\hline Mean age, yrs & $59.1(13.5)$ & $57.6(14.2)$ & $61.8(12.1)$ & 0.204 \\
\hline Sex & & & & 0.637 \\
\hline Female & $29(38.7 \%)$ & $18(62.1 \%)$ & $11(37.9 \%)$ & \\
\hline Male & $46(61.3 \%)$ & $31(67.4 \%)$ & $15(32.6 \%)$ & \\
\hline Median KPS score & 70 & 70 & 60 & 0.001 \\
\hline Mean CCl score & $8.7(2.3)$ & $8.5(1.9)$ & $9.3(2.9)$ & 0.211 \\
\hline \multicolumn{5}{|l|}{ SINS } \\
\hline 7 & $3(4.0 \%)$ & $3(100.0 \%)$ & $0(0.0 \%)$ & 0.547 \\
\hline 8 & $7(9.3 \%)$ & $6(85.7 \%)$ & $1(14.3 \%)$ & 0.410 \\
\hline 9 & $7(9.3 \%)$ & $6(85.7 \%)$ & $1(14.3 \%)$ & 0.410 \\
\hline 10 & $12(16.0 \%)$ & $10(83.3 \%)$ & $2(16.7 \%)$ & 0.198 \\
\hline 11 & $17(22.7 \%)$ & $11(64.7 \%)$ & $6(35.3 \%)$ & 0.951 \\
\hline 12 & $29(38.7 \%)$ & $13(44.8 \%)$ & $16(55.2 \%)$ & 0.003 \\
\hline Location & & & & 0.655 \\
\hline Junctional & $41(54.7 \%)$ & $27(65.9 \%)$ & $14(34.1 \%)$ & \\
\hline Mobile & $13(17.3 \%)$ & $9(69.2 \%)$ & $4(30.8 \%)$ & \\
\hline Semirigid & $19(25.3 \%)$ & $11(57.9 \%)$ & $8(42.1 \%)$ & \\
\hline Rigid & $2(2.7 \%)$ & $2(100.0 \%)$ & $0(0.0 \%)$ & \\
\hline Pain & & & & 0.088 \\
\hline Mechanical & $65(86.7 \%)$ & $40(61.5 \%)$ & $25(38.5 \%)$ & \\
\hline Oncological & $2(2.7 \%)$ & $1(50.0 \%)$ & $1(50.0 \%)$ & \\
\hline None & $8(10.7 \%)$ & $8(100.0 \%)$ & $0(0.0 \%)$ & \\
\hline Lesion type & & & & 0.015 \\
\hline Lytic & $19(25.3 \%)$ & $8(42.1 \%)$ & $11(57.9 \%)$ & \\
\hline Mixed & $35(46.7 \%)$ & $23(65.7 \%)$ & $12(34.3 \%)$ & \\
\hline Blastic & $21(28.0 \%)$ & $18(85.7 \%)$ & $3(14.3 \%)$ & \\
\hline Alignment & & & & 0.711 \\
\hline Subluxation & $0(0.0 \%)$ & $0(0.0 \%)$ & $0(0.0 \%)$ & \\
\hline Kyphosis & $9(12.0 \%)$ & $5(55.6 \%)$ & $4(44.4 \%)$ & \\
\hline None & $66(88.0 \%)$ & $44(66.7 \%)$ & $22(33.3 \%)$ & \\
\hline Vertebral body involvement & & & & 0.033 \\
\hline$>50 \%$ collapse & $19(25.3 \%)$ & $14(73.7 \%)$ & $5(26.3 \%)$ & \\
\hline$<50 \%$ collapse & $25(33.3 \%)$ & $14(56.0 \%)$ & $11(44.0 \%)$ & \\
\hline$>50 \%$ involvement & $17(22.7 \%)$ & $8(47.1 \%)$ & $9(52.9 \%)$ & \\
\hline None & $14(18.7 \%)$ & $13(92.9 \%)$ & $1(7.1 \%)$ & \\
\hline Posterior involvement & & & & 0.929 \\
\hline Bilateral & $66(88.0 \%)$ & $43(65.2 \%)$ & $23(34.8 \%)$ & \\
\hline Unilateral & $9(12.0 \%)$ & $6(66.7 \%)$ & $3(33.0 \%)$ & \\
\hline None & $0(0.0 \%)$ & $0(0.0 \%)$ & $0(0.0 \%)$ & \\
\hline
\end{tabular}

Values represent the number of patients (\%) or mean (SD) unless stated otherwise.

eral, unilateral, or none) $(\mathrm{p}=0.033)$. Patients who initially underwent radiation therapy but subsequently converted to surgery underwent intervention for 1) spinal cord compression, 2) severe intractable pain, or 3) tumor growth despite radiation and chemotherapy with impending cord compression. The mean time from radiation to conversion to surgical treatment was $17.77 \pm 25.16$ months. Indications for surgery, types of surgery, and time to surgery are summarized in Table 4 for both patients who were initially treated with radiation and those initially treated with surgery.

A subsequent multivariate backward likelihood binary regression analysis revealed SINS $>11$ (OR 8.09, CI $1.96-33.4, p=0.004$ ) was independently associated with an increased risk of surgical intervention. The model also revealed that an increased KPS score (OR 0.94, CI $0.89-0.98, \mathrm{p}=0.008$ ) was associated with a reduced risk of surgical intervention. However, KPS score was not sig- 
TABLE 4. Indications for surgery in patients with SINS 7-12 initially undergoing radiation therapy versus upfront surgery

\begin{tabular}{|c|c|}
\hline Variable & Value \\
\hline \multicolumn{2}{|l|}{ Indication for surgery following initial RT $(n=26)$} \\
\hline Spinal cord compression & $9(34.62 \%)$ \\
\hline Severe intractable pain & $7(26.92 \%)$ \\
\hline $\begin{array}{l}\text { Treatment-resistant tumor growth w/ impending } \\
\text { cord compression }\end{array}$ & $10(38.46 \%)$ \\
\hline \multicolumn{2}{|l|}{ Surgical treatment } \\
\hline Vertebroplasty & $2(7.69 \%)$ \\
\hline Tumor debulking & $6(23.07 \%)$ \\
\hline Fixation & $18(69.23 \%)$ \\
\hline Mean time from initial RT to surgery (mos) & $17.77(25.16)$ \\
\hline \multicolumn{2}{|l|}{ Indication for upfront surgery $(n=98)$} \\
\hline Spinal cord compression & $43(43.87 \%)$ \\
\hline Severe intractable pain & $37(37.76 \%)$ \\
\hline Tumor growth w/ impending cord compression & $18(18.37 \%)$ \\
\hline \multicolumn{2}{|l|}{ Surgical treatment } \\
\hline Vertebroplasty & $3(3.06 \%)$ \\
\hline Tumor debulking & $38(38.78 \%)$ \\
\hline Fixation & $57(58.16 \%)$ \\
\hline
\end{tabular}

$\mathrm{RT}=$ radiation therapy.

Values represent the number of patients (\%) unless stated otherwise. Mean values are presented as the mean (SD).

nificantly correlated with SINS (coefficient $=-0.08, \mathrm{p}=$ $0.40)$. Solving the logistic regression model, $P=e^{-8+0.678 x /}$ $\left(1+\mathrm{e}^{-8+0.678 \mathrm{x}}\right)$, as a function of SINS for a probability of $50 \%$ provided a SINS cutoff of 11.8. Patients with SINS $<11$ required surgery $17.1 \%$ of the time compared with $50.6 \%$ in patients with SINS $\geq 11(\mathrm{p}=0.001)$.

RPA was then utilized to further evaluate prognosticators of surgical intervention and clinically relevant cutoffs. In a patient-specific analysis, RPA identified a SINS cutoff of $>10$ as having a $47.8 \%$ incidence of surgical intervention and SINS $\leq 10$ as having a $13.8 \%$ incidence of surgical intervention (Fig. 1A). Similarly, a per-lesion RPA identified a cutoff of SINS 10. On the per-lesion analysis, lesions with SINS $\leq 10$ had a $24.2 \%$ incidence of surgical intervention, but SINS $>10$ had a 50.4\% incidence of surgical intervention (Fig. 1B). RPA per-patient subanalysis identified clinical KPS score cutoffs as well. The first de- cision node identified in the RPA was a KPS cutoff of 60 . Patients with a KPS score $>60$ had a $17.6 \%$ incidence of surgical intervention as compared with $70.8 \%$ in patients with a KPS score $\leq 60$. In patients with a KPS score $>60$, patients with SINS > 10 had a $32.1 \%$ incidence of surgical intervention, relative to a $0.0 \%$ incidence in patients with SINS $\leq 10$ (Fig. 3). RPA analysis did not identify a SINS cutoff among patients with a KPS score $<60$.

\section{Treatment, Perioperative, 30-Day, and 90-Day Outcomes}

A comparison of treatment, perioperative, 30-day, and 90-day outcomes between patients with and those without surgical intervention is shown in Table 1. An MR image of a spine lesion with potential instability is shown in Fig. 4. Patients with a surgical intervention were more likely to have undergone chemotherapy ( $46.2 \%$ vs $6.1 \%$, p < 0.001$)$, kyphoplasty $(11.5 \%$ vs $0.0 \%, \mathrm{p}=0.015)$, and vertebroplasty $(11.5 \%$ vs $0.0 \%, p=0.015)$. Similarly, patients with surgical intervention had a higher incidence of perioperative complications ( $42.3 \%$ vs $18.4 \%, \mathrm{p}=0.026)$, 30-day readmission $(15.4 \%$ vs $2.0 \%, \mathrm{p}=0.046)$, and 90 -day readmission $(23.1 \%$ vs $2.0 \%, \mathrm{p}=0.006)$. The 30 - and 90 -day reoperation rates for patients who underwent stabilization surgery were both $15.4 \%$. A multivariate backward likelihood binary regression analysis revealed surgical intervention to be independently associated with development of a perioperative complication (OR 2.96, CI 1.01-8.71, p $=0.048$ ).

\section{Discussion}

Although the SINS classification by Fisher et al. ${ }^{3}$ has defined stable and unstable spinal metastatic lesions, there is no clear consensus within the potentially unstable category (SINS 7-12). Our study is the largest to date. We focused only on patients with potentially unstable SINS who were initially managed nonoperatively, quantified the proportion of patients who required eventual surgical stabilization, and attempted to determine if there exists an optimal cutoff above which a potentially unstable lesion would be more likely to require stabilization. Previous studies have suggested that a SINS of 10 or greater may represent a more appropriate score for stabilization as opposed to a SINS of 13 or greater. ${ }^{19}$ The impetus to further define a score necessitating stabilization arises from the need to treat pain as well as prevent adverse spinal events which could result in catastrophic neurological complications. Studies have correlated metastatic disease causing

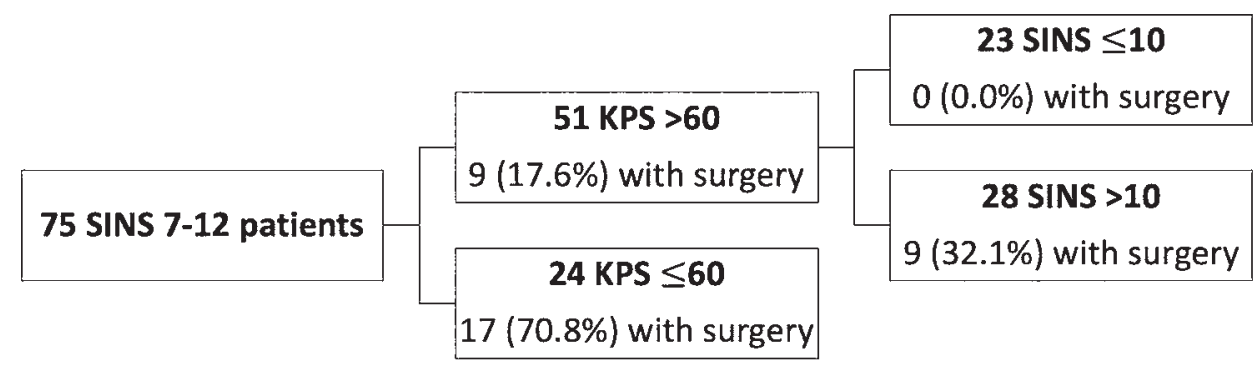

FIG. 3. KPS score recursive partition subanalysis. 


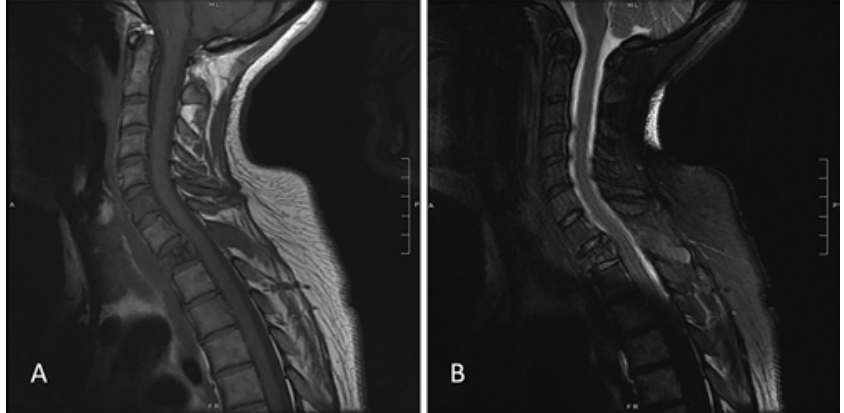

FIG. 4. Sagittal T1-weighted (A) and T2-weighted (B) MR images of a potentially unstable spine.

spinal instability with adverse spinal events as well as SINS ability to predict those events. ${ }^{20-23}$ Lam et al. reported that SINS $\geq 11$ resulted in more than 2.5 times the risk of adverse spinal events following stereotactic body radiotherapy compared with patients with SINS $\leq 10 .{ }^{22}$ Versteeg et al. performed a multicenter study of 1509 patients and compared SINS of operative and nonoperative patients. ${ }^{24}$ They identified a mean SINS of 7.2 in the nonoperative group and 10.7 in the operative group. These results are consistent with our findings as well as those of Pennington et al. ${ }^{19}$

In a retrospective review, Pennington et al. ${ }^{19}$ analyzed 38 patients with SINS indicating potential instability and suggested that lesions with a SINS $<10$ may not require stabilization. In their study, patients who underwent fusion had a higher mean SINS (11 vs 8), higher proportion of lytic lesions, higher mean KPS score, and more extensive epidural involvement. The authors reported an optimal SINS cutoff of 10 , with patients with SINS $\geq 10$ having $>$ $50 \%$ odds of undergoing stabilization surgery. This supports the optimal SINS cutoff of 10, above which surgical stabilization may be appropriate, that was also identified in our study. In contrast to Pennington et al., however, we found that patients who underwent stabilization surgery had lower rather than higher KPS scores, and reduced KPS score was associated with increased risk of surgical intervention on multivariable analysis. This could potentially be reflective of increased spine instability and pain contributing to lower KPS scores, translating to a need for surgical intervention. The association of higher KPS score and increased risk of undergoing stabilization surgery observed by Pennington et al. may be reflective of selection bias as patients with higher KPS scores may be offered surgery more readily due to their higher functioning status and ability to tolerate an operation.

Because mechanical instability represents a main indication for surgery in spinal oncology patients, clarifying the role of surgical intervention in the potentially unstable SINS category can be helpful. Studies have reported up to $75 \%$ of patients appear in the potentially unstable category, further underscoring the need for additional analysis of this population. ${ }^{19}$ Dosani et al. found that patients with SINS of 7-12 were more likely to undergo surgical consultation for consideration of stabilization. ${ }^{25}$ Our findings of higher rates of surgical intervention correlating with higher SINS in the potentially unstable group is consistent with the lit- erature, as is the finding of a suggested threshold of SINS of 10. Surgeons may be more likely to offer these patients stabilization given the possible instability and possible impending collapse of the spine. In patients who were initially chosen to undergo radiation only, the main reasons were 1) the patients did not meet the Patchell criteria for surgery, 2) there was no gross instability of the spine based on radiographic imaging, or 3 ) the patients were simply too ill to undergo surgery. In our study, some patients with SINS 7-12 who initially received radiation therapy were subsequently converted to surgery. Progressive, incapacitating pain was a driver for surgery. Spinal cord compression with resultant neurological changes would have been another impetus for surgery. Asymptomatic patients were converted to surgery if there was radiographic follow-up showing spinal canal stenosis, continued tumor growth despite chemotherapy or radiation, or impending spinal cord compression.

\section{Study Limitations}

There are limitations to this study. First, it is a retrospective, single-institution review, and larger, multiinstitutional studies would be useful to validate our findings. There may be selection bias in this study in that patients with lower KPS scores may be in more pain and may have been more likely to undergo surgery. This may have been independent of SINS score, but it certainly may also have been correlated with the SINS and pain from the spine. There are other factors which should be considered when interpreting the data. The overall patient context is important. Patients with a SINS $\geq 12$ may not undergo surgical stabilization if their prognosis is poor. Alternatively, patients with stable lesions may undergo surgery for spinal cord compression issues, not for stability issues. In order to help minimize these confounders, we performed perpatient and per-lesion analyses.

It is also important to acknowledge the significantly higher rate of complications and readmissions observed in the surgical subgroup, as this could potentially be due to baseline differences. For example, it is plausible that the higher rate of chemotherapy in patients undergoing surgery could lead to related complications such as fever, neutropenia, and anemia. Of the 26 patients requiring surgical treatment, $30.7 \%$ of the patients developed a complication or required readmission for medical causes not directly related to the surgery (e.g., pneumonia, arrhythmia, and anemia). The rest of the patients developed at least one complication directly related to their surgery (e.g., hematoma evacuation, surgical site infection, or wound dehiscence). These data support the notion that most of the complications and readmissions were a sequalae of surgery.

\section{Conclusions}

In patients with potentially unstable SINS, lesions with scores $\geq 10$ may be at increased risk for mechanical instability requiring stabilizing surgery. KPS score was not associated with SINS. RPA found a significant SINS cutoff of 10 for patients with KPS scores > 60; however, no similar cutoff was observed in patients with KPS scores $\leq 60$. 


\section{References}

1. Patchell RA, Tibbs PA, Regine WF, et al. Direct decompressive surgical resection in the treatment of spinal cord compression caused by metastatic cancer: a randomised trial. Lancet. 2005;366(9486):643-648.

2. Laufer I, Rubin DG, Lis E, et al. The NOMS framework: approach to the treatment of spinal metastatic tumors. Oncologist. 2013;18(6):744-751.

3. Fisher CG, DiPaola CP, Ryken TC, et al. A novel classification system for spinal instability in neoplastic disease: an evidence-based approach and expert consensus from the Spine Oncology Study Group. Spine (Phila Pa 1976). 2010; 35(22):E1221-E1229.

4. Versteeg AL, Verlaan JJ, Sahgal A, et al. The Spinal Instability Neoplastic Score: impact on oncologic decision-making. Spine (Phila Pa 1976). 2016;41(suppl 20):S231-S237.

5. Hussain I, Barzilai O, Reiner AS, et al. Spinal Instability Neoplastic Score component validation using patient-reported outcomes. J Neurosurg Spine. 2019;30(4):432-438.

6. Versteeg AL, Sahgal A, Rhines LD, et al. Health related quality of life outcomes following surgery and/or radiation for patients with potentially unstable spinal metastases. Spine J. 2021;21(3):492-499.

7. Hussain I, Barzilai O, Reiner AS, et al. Patient-reported outcomes after surgical stabilization of spinal tumors: symptombased validation of the Spinal Instability Neoplastic Score (SINS) and surgery. Spine J. 2018;18(2):261-267.

8. Fisher CG, Schouten R, Versteeg AL, et al. Reliability of the Spinal Instability Neoplastic Score (SINS) among radiation oncologists: an assessment of instability secondary to spinal metastases. Radiat Oncol. 2014;9:69.

9. Fisher CG, Versteeg AL, Schouten R, et al. Reliability of the spinal instability neoplastic scale among radiologists: an assessment of instability secondary to spinal metastases. AJR Am J Roentgenol. 2014;203(4):869-874.

10. Arana E, Kovacs FM, Royuela A, et al. Spine Instability Neoplastic Score: agreement across different medical and surgical specialties. Spine J. 2016;16(5):591-599.

11. Campos M, Urrutia J, Zamora T, et al. The Spine Instability Neoplastic Score: an independent reliability and reproducibility analysis. Spine J. 2014;14(8):1466-1469.

12. Pennington Z, Ahmed AK, Cottrill E, et al. Intra- and interobserver reliability of the Spinal Instability Neoplastic Score system for instability in spine metastases: a systematic review and meta-analysis. Ann Transl Med. 2019;7(10):218.

13. Lee SH, Tatsui CE, Ghia AJ, et al. Can the spinal instability neoplastic score prior to spinal radiosurgery predict compression fractures following stereotactic spinal radiosurgery for metastatic spinal tumor? A post hoc analysis of prospective phase II single-institution trials. J Neurooncol. 2016;126(3): 509-517.

14. Shi DD, Hertan LM, Lam TC, et al. Assessing the utility of the spinal instability neoplastic score (SINS) to predict fracture after conventional radiation therapy (RT) for spinal metastases. Pract Radiat Oncol. 2018;8(5):e285-e294.

15. Cavalcante RA, Fernandes YB, Marques RA, et al. Is there a correlation between the spinal instability neoplastic score and mechanical pain in patients with metastatic spinal cord compression? A prospective cohort study. J Craniovertebr Junction Spine. 2017;8(3):187-192.

16. Dakson A, Leck E, Brandman DM, Christie SD. The clinical utility of the Spinal Instability Neoplastic Score (SINS) system in spinal epidural metastases: a retrospective study. Spinal Cord. 2020;58(8):892-899.
17. de Oliveira MF, Rotta JM, Botelho RV. Is there a relationship between spinal instability in neoplastic disease and Tokuhashi scoring system? Neurosurg Rev. 2016;39(3):519-524.

18. Masuda K, Ebata K, Yasuhara Y, et al. Outcomes and prognosis of neurological decompression and stabilization for spinal metastasis: is assessment with the Spinal Instability Neoplastic Score useful for predicting surgical results? Asian Spine J. 2018;12(5):846-853.

19. Pennington Z, Ahmed AK, Westbroek EM, et al. SINS score and stability: evaluating the need for stabilization within the uncertain category. World Neurosurg. 2019;128:e1034-e1047.

20. Aiba H, Kimura T, Yamagami T, et al. Prediction of skeletalrelated events in patients with non-small cell lung cancer. Support Care Cancer. 2016;24(8):3361-3367.

21. Chang SY, Ha JH, Seo SG, et al. Prognosis of single spinal metastatic tumors: predictive value of the Spinal Instability Neoplastic Score system for spinal adverse events. Asian Spine J. 2018;12(5):919-926.

22. Lam TC, Uno H, Krishnan M, et al. Adverse outcomes after palliative radiation therapy for uncomplicated spine metastases: role of spinal instability and single-fraction radiation therapy. Int J Radiat Oncol Biol Phys. 2015;93(2):373-381.

23. Sahgal A, Atenafu EG, Chao S, et al. Vertebral compression fracture after spine stereotactic body radiotherapy: a multi-institutional analysis with a focus on radiation dose and the Spinal Instability Neoplastic Score. J Clin Oncol. 2013; 31(27):3426-3431.

24. Versteeg AL, van der Velden JM, Verkooijen HM, et al. The effect of introducing the Spinal Instability Neoplastic Score in routine clinical practice for patients with spinal metastases. Oncologist. 2016;21(1):95-101.

25. Dosani M, Lucas S, Wong J, et al. Impact of the Spinal Instability Neoplastic Score on surgical referral patterns and outcomes. Curr Oncol. 2018;25(1):53-58.

\section{Disclosures}

Dr. Mummaneni: consultant for DePuy Synthes, Globus, and Stryker; direct stock ownership in Spinicity/ISD; support of nonstudy-related clinical or research effort from AO Spine, NREF, and ISSG; honoraria from AO Spine; and royalties from DePuy Synthes, Thieme Publishers, and Springer Publishers. Dr. Berven: consultant for Medtronic, Globus, Innovasis, Integrity, Stryker, and Camber; ownership in Green Sun and Providence; and royalties from Stryker. Dr. Chou: consultant for and royalties from Globus.

\section{Author Contributions}

Conception and design: Chou, Vargas, Lockney, Mummaneni, Berven. Acquisition of data: Chou, Vargas, Mummaneni, Rivera, Tan, Jamieson, Mahmoudieh, Berven, Braunstein. Analysis and interpretation of data: Chou, Vargas, Lockney, Haddad. Drafting the article: Chou, Vargas, Lockney, Rivera. Critically revising the article: Chou, Vargas, Lockney, Mummaneni, Haddad, Rivera, Tan, Braunstein. Reviewed submitted version of manuscript: Chou, Vargas, Mummaneni, Haddad, Rivera, Berven, Braunstein. Statistical analysis: Haddad. Study supervision: Chou, Lockney, Mummaneni.

\section{Correspondence}

Dean Chou: University of California, San Francisco, CA. dean. chou@ucsf.edu. 